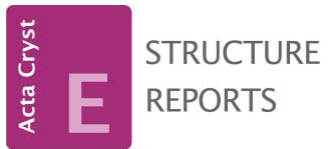

OPEN $\curvearrowright$ ACCESS

ISSN $1600-5368$

\section{Crystal structure of 2-cyano-N'-(cyclo- hexylidene)acetohydrazide}

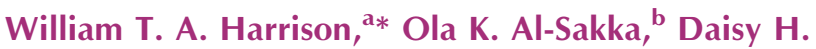 \\ Fleita, ${ }^{\text {b }}$ Amina Saleh ${ }^{\mathrm{b}, \mathrm{c}}$ and Sara Salem ${ }^{\mathrm{b}}$
}

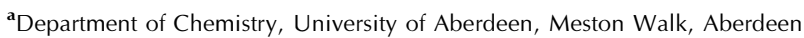
AB24 3UE, Scotland, 'bepartment of Chemistry, American University in Cairo, PO Box 74, New Cairo 11835, Egypt, and Institute of Chemistry and Chemical Engineering, École Polytechnique Fédérale de Lausanne, Station 6, CH-1015 Lausanne, Switzerland. *Correspondence e-mail: w.harrison@abdn.ac.uk

Received 2 April 2014; accepted 24 April 2014

Edited by H. Stoeckli-Evans, University of Neuchâtel, Switzerland

In the title compound, $\mathrm{C}_{9} \mathrm{H}_{13} \mathrm{~N}_{3} \mathrm{O}$, the cyclohexylidene ring adopts a chair conformation and the bond-angle sum at the $\mathrm{C}$ atom linked to the $\mathrm{N}$ atom is $359.6^{\circ}$. The cyanoacetohydrazide grouping is close to planar (r.m.s. deviation for the non-H atoms $=0.031 \AA$ ) and subtends a dihedral angle of $64.08(4)^{\circ}$ with the four $\mathrm{C}$ atoms forming the seat of the chair. The $\mathrm{C}=\mathrm{O}$ and $\mathrm{N}-\mathrm{H}$ groups are in a syn conformation $(\mathrm{O}-\mathrm{C}-\mathrm{N}-\mathrm{H}=$ $-5^{\circ}$ ). In the crystal, inversion dimers linked by pairs of $\mathrm{N}-$ $\mathrm{H} \cdots \mathrm{O}$ hydrogen bonds generate $R_{2}^{2}(8)$ loops; this dimer linkage is reinforced by a pair of $\mathrm{C}-\mathrm{H} \cdots \mathrm{O}$ interactions, which generate $R_{2}^{2}(14)$ loops. The dimers are linked by $\mathrm{C}-\mathrm{H} \cdots \mathrm{N}_{\mathrm{c}}(\mathrm{c}$ = cyanide) interactions into [100] ladders, which feature $C(4)$ chains and $R_{4}^{4}(20)$ loops.

Keywords: crystal structure; hydrazide; cyclohexylidene; inversion dimer.

CCDC reference: 1004279

\section{Related literature}

For background to the role of hydrazides as potential anticancer agents, see: Sechi et al. (2008); Manivel et al. (2009); Mohareb et al. (2011).

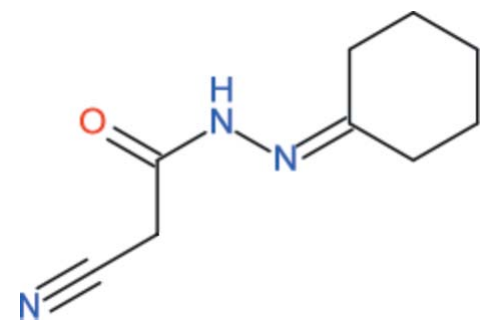

\section{Experimental}

2.1. Crystal data

$\mathrm{C}_{9} \mathrm{H}_{13} \mathrm{~N}_{3} \mathrm{O}$

$M_{r}=179.22$

Triclinic, $P \overline{1}$

$a=4.8420(2) \AA$

$b=9.7407$ (7) $\AA$

$c=10.7071(8) \AA$

$\alpha=73.917$ (9)

$\beta=82.819(10)^{\circ}$

$$
\begin{aligned}
& \gamma=75.980(9)^{\circ} \\
& V=469.87(5) \AA^{3} \\
& Z=2 \\
& \text { Mo } K \alpha \text { radiation } \\
& \mu=0.09 \mathrm{~mm}^{-1} \\
& T=100 \mathrm{~K} \\
& 0.13 \times 0.12 \times 0.04 \mathrm{~mm}
\end{aligned}
$$

\subsection{Data collection \\ Rigaku Mercury CCD diffractometer 6176 measured reflections}

2136 independent reflections 1789 reflections with $I>2 \sigma(I)$ $R_{\text {int }}=0.024$

\subsection{Refinement}

$R\left[F^{2}>2 \sigma\left(F^{2}\right)\right]=0.035$

$w R\left(F^{2}\right)=0.101$

$S=1.08$

2136 reflections

121 parameters
$\mathrm{H}$ atoms treated by a mixture of independent and constrained refinement
$\Delta \rho_{\max }=0.29{\mathrm{e} \AA^{-3}}^{-3}$
$\Delta \rho_{\min }=-0.19{\mathrm{e} \AA^{-3}}^{-3}$

Table 1

Hydrogen-bond geometry $\left(\AA{ }^{\circ}\right)$.

\begin{tabular}{lllll}
\hline$D-\mathrm{H} \cdots A$ & $D-\mathrm{H}$ & $\mathrm{H} \cdots A$ & $D \cdots A$ & $D-\mathrm{H} \cdots A$ \\
\hline $\mathrm{N}^{2}-\mathrm{H} 1 \cdots \mathrm{O} 1^{\mathrm{i}}$ & $0.900(14)$ & $2.052(15)$ & $2.9399(12)$ & $168.4(11)$ \\
$\mathrm{C}^{\mathrm{i}}-\mathrm{H} 6 B \cdots \mathrm{O} 1^{\mathrm{i}}$ & 0.99 & 2.32 & $3.2736(13)$ & 161 \\
$\mathrm{C} 8-\mathrm{H} 8 B \cdots \mathrm{N}^{3}{ }^{\mathrm{i}}$ & 0.99 & 2.41 & $3.3783(14)$ & 165
\end{tabular}

Symmetry codes: (i) $-x,-y+1,-z$; (ii) $x+1, y, z$.

Data collection: CrystalClear (Rigaku, 2012); cell refinement: CrystalClear; data reduction: CrystalClear; program(s) used to solve structure: SHELXS97 (Sheldrick, 2008); program(s) used to refine structure: SHELXL97 (Sheldrick, 2008); molecular graphics: ORTEP-3 for Windows (Farrugia, 2012); software used to prepare material for publication: SHELXL97.

\section{Acknowledgements}

The authors thank the American University in Cairo for financial support.

Supporting information for this paper is available from the IUCr electronic archives (Reference: SU0002).

\section{References}

Farrugia, L. J. (2012). J. Appl. Cryst. 45, 849-854.

Manivel, P., Mohana Roopan, S., Sathish Kumar, R. \& Nawazkhan, F. (2009). J. Chil. Chem. Soc. 54, 183-185.

Mohareb, R. M., Fleita, D. H. \& Sakka Ola, K. (2011). Molecules, 16, 16-27. Rigaku (2012). CrystalClear. Rigaku Inc., Tokyo, Japan.

Sechi, M., Azeena, U., Delussu, M. P., Dallochio, R., Dessi, A., Cosseddu, A., Pala, N. \& Neamati, N. (2008). Molecules, 13, 2442-2461.

Sheldrick, G. M. (2008). Acta Cryst. A64, 112-122. 


\section{supporting information}

Acta Cryst. (2014). E70, o886 [doi:10.1107/S1600536814009350]

\section{Crystal structure of 2-cyano-N'-(cyclohexylidene)acetohydrazide}

\section{William T. A. Harrison, Ola K. Al-Sakka, Daisy H. Fleita, Amina Saleh and Sara Salem}

\section{S1. Experimental}

Cyclohexanone $(0.98 \mathrm{~g}, 0.01 \mathrm{~mol})$ was added to a solution of cyanoacetylhydrazine $(0.99 \mathrm{~g}, 0.01 \mathrm{~mol})$ in 1,4-dioxane (20 $\mathrm{ml}$ ). The mixture was heated under reflux for $2 \mathrm{~h}$ and then poured into a beaker containing an ice/water mixture: the solid product was collected by filtration. Yellow slabs of the title compound were obtained by slow evaporation of an ethanol solution.

\section{S2. Refinement}

The $\mathrm{N}$-bound $\mathrm{H}$ atom was located in a difference map and its position was freely refined. The $\mathrm{C}$-bound $\mathrm{H}$ atoms were placed in idealized locations $(\mathrm{C}-\mathrm{H}=0.99 \AA)$ and refined as riding atoms. The constraint $U_{\text {iso }}(\mathrm{H})=1.2 U_{\text {eq }}($ carrier $)$ was applied in all cases.

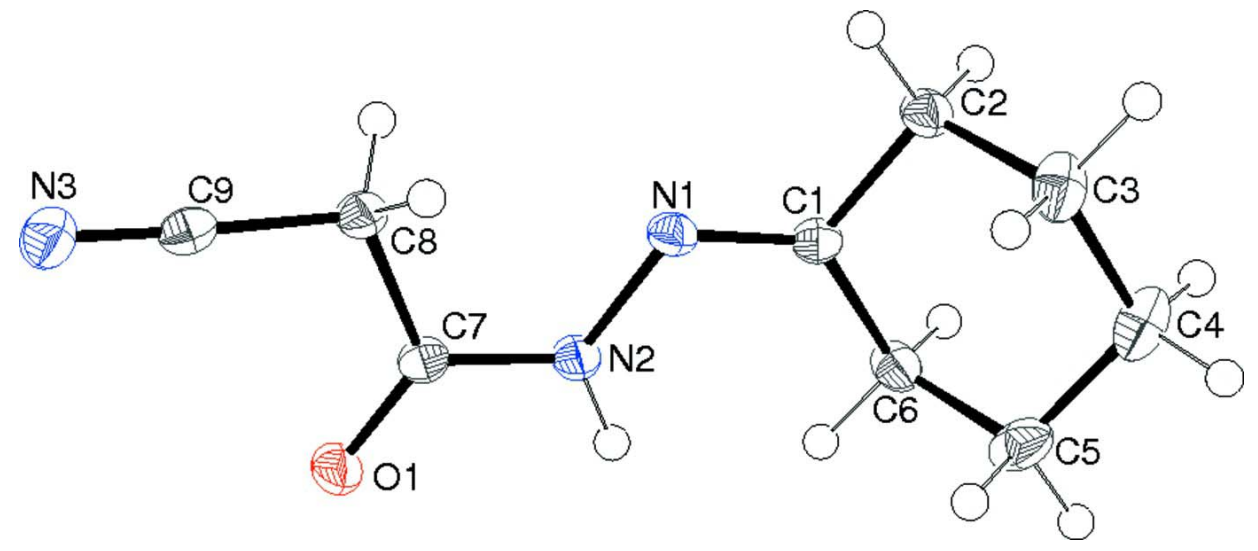

\section{Figure 1}

The molecular structure of the title compound showing 50\% displacement ellipsoids. 


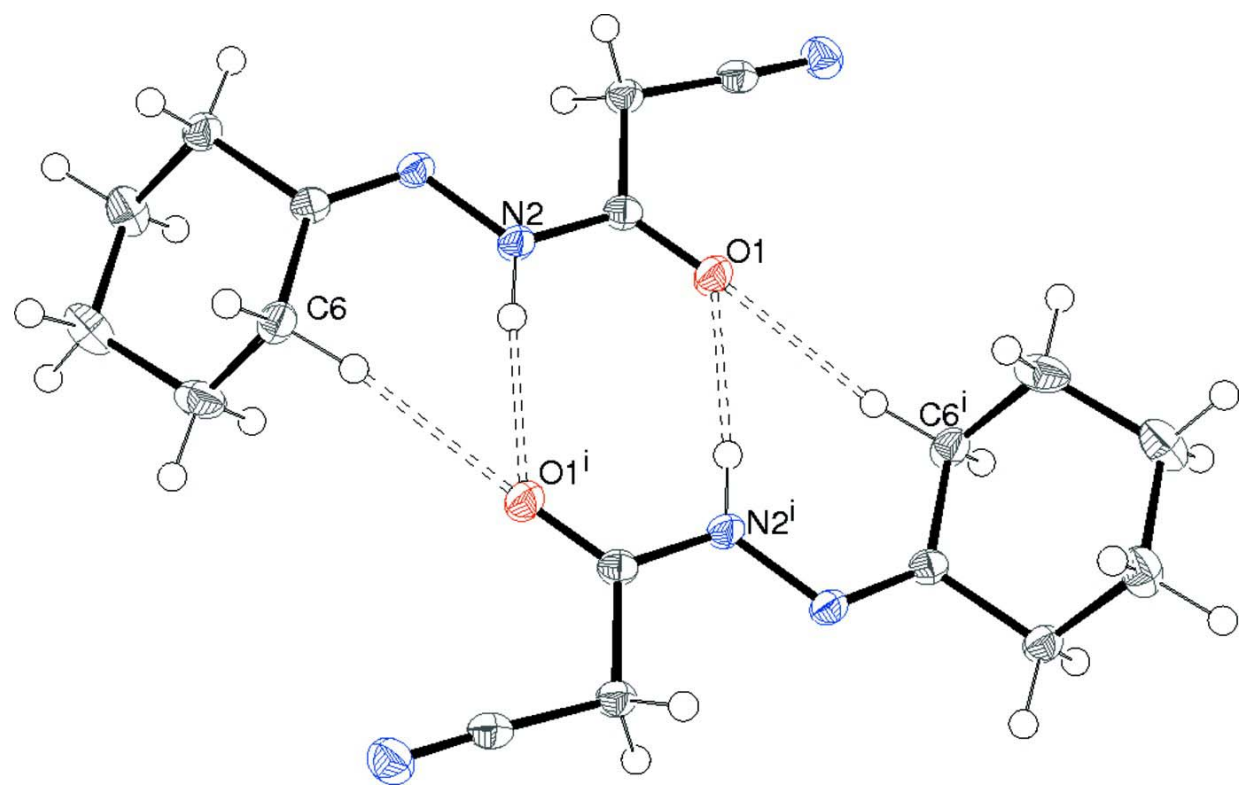

\section{Figure 2}

An inversion dimer in the crystal of the title compound, with $\mathrm{N}-\mathrm{H} \cdots \mathrm{O}$ and $\mathrm{C}-\mathrm{H} \cdots \mathrm{O}$ hydrogen bonds indicated by double-dashed lines. Symmetry code: (i) $-\mathrm{x}, 1-\mathrm{y},-\mathrm{z}$.

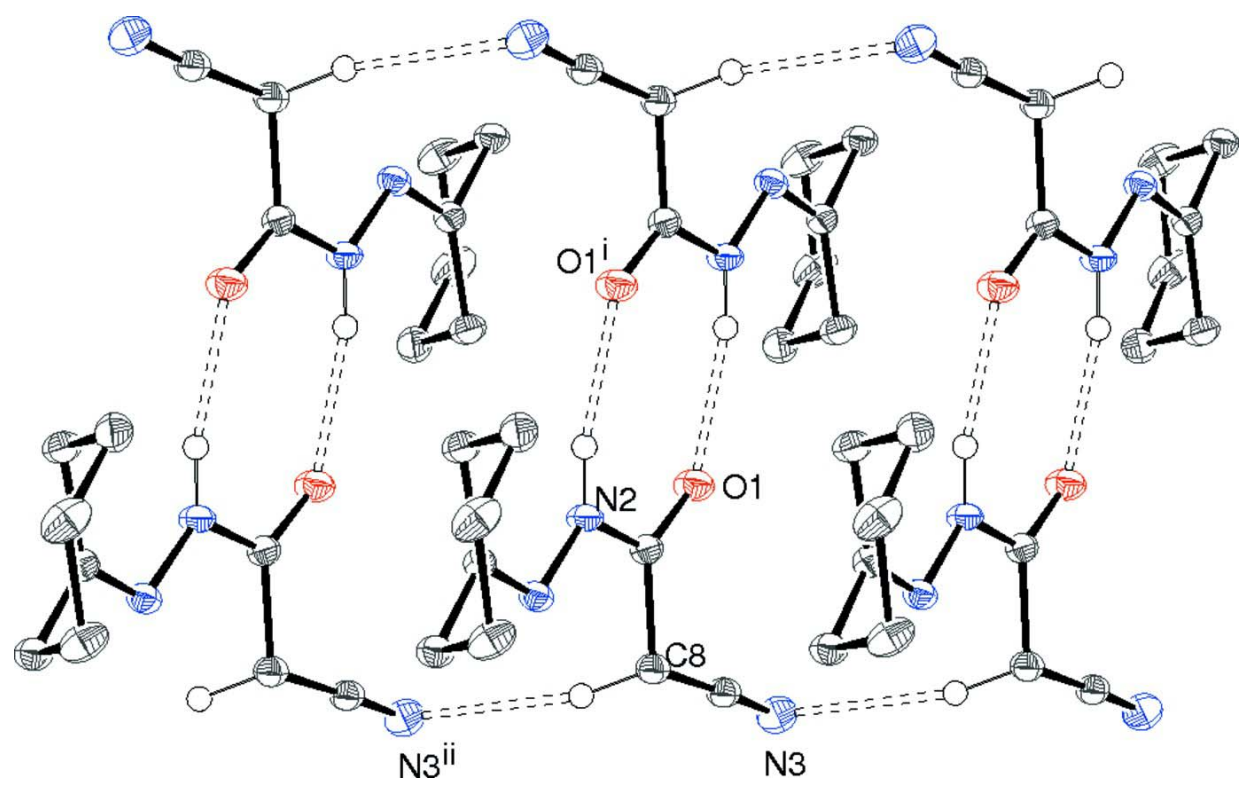

\section{Figure 3}

Part of a [100] double chain in the crystal of the title compound, with hydrogen bonds indicated by double-dashed lines. Symmetry codes: (i) $-\mathrm{x}, 1-\mathrm{y},-\mathrm{z}$; (ii) $1+x, y, z$.

\section{2-Cyano-N'-(cyclohexylidene)acetohydrazide}

Crystal data

$\mathrm{C}_{9} \mathrm{H}_{13} \mathrm{~N}_{3} \mathrm{O}$

$M_{r}=179.22$

Triclinic, $P \overline{1}$
Hall symbol: -P 1

$a=4.8420$ (2) $\AA$

$b=9.7407$ (7) $\AA$ 


$$
\begin{aligned}
& c=10.7071(8) \AA \\
& \alpha=73.917(9)^{\circ} \\
& \beta=82.819(10)^{\circ} \\
& \gamma=75.980(9)^{\circ} \\
& V=469.87(5) \AA^{3} \\
& Z=2 \\
& F(000)=192 \\
& D_{\mathrm{x}}=1.267 \mathrm{Mg} \mathrm{m}^{-3}
\end{aligned}
$$

Data collection

Rigaku Mercury CCD diffractometer

Radiation source: fine-focus sealed tube Graphite monochromator $\omega$ scans

6176 measured reflections

2136 independent reflections

\section{Refinement}

Refinement on $F^{2}$

Least-squares matrix: full

$R\left[F^{2}>2 \sigma\left(F^{2}\right)\right]=0.035$

$w R\left(F^{2}\right)=0.101$

$S=1.08$

2136 reflections

121 parameters

0 restraints

Primary atom site location: structure-invariant direct methods
Mo $K \alpha$ radiation, $\lambda=0.71073 \AA$

Cell parameters from 5790 reflections

$\theta=2.6-27.5^{\circ}$

$\mu=0.09 \mathrm{~mm}^{-1}$

$T=100 \mathrm{~K}$

Cut slab, yellow

$0.13 \times 0.12 \times 0.04 \mathrm{~mm}$

1789 reflections with $I>2 \sigma(I)$

$R_{\text {int }}=0.024$

$\theta_{\max }=27.5^{\circ}, \theta_{\min }=2.6^{\circ}$

$h=-6 \rightarrow 5$

$k=-12 \rightarrow 11$

$l=-13 \rightarrow 13$

Secondary atom site location: difference Fourier map

Hydrogen site location: inferred from neighbouring sites

$\mathrm{H}$ atoms treated by a mixture of independent and constrained refinement

$w=1 /\left[\sigma^{2}\left(F_{\mathrm{o}}^{2}\right)+(0.0528 P)^{2}+0.0762 P\right]$ where $P=\left(F_{\mathrm{o}}^{2}+2 F_{\mathrm{c}}^{2}\right) / 3$

$(\Delta / \sigma)_{\max }<0.001$

$\Delta \rho_{\max }=0.29 \mathrm{e} \AA^{-3}$

$\Delta \rho_{\min }=-0.19 \mathrm{e} \AA^{-3}$

\section{Special details}

Geometry. All e.s.d.'s (except the e.s.d. in the dihedral angle between two 1.s. planes) are estimated using the full covariance matrix. The cell e.s.d.'s are taken into account individually in the estimation of e.s.d.'s in distances, angles and torsion angles; correlations between e.s.d.'s in cell parameters are only used when they are defined by crystal symmetry. An approximate (isotropic) treatment of cell e.s.d.'s is used for estimating e.s.d.'s involving 1.s. planes.

Refinement. Refinement of $F^{2}$ against ALL reflections. The weighted $R$-factor $w R$ and goodness of fit $S$ are based on $F^{2}$, conventional $R$-factors $R$ are based on $F$, with $F$ set to zero for negative $F^{2}$. The threshold expression of $F^{2}>\sigma\left(F^{2}\right)$ is used only for calculating $R$-factors(gt) etc. and is not relevant to the choice of reflections for refinement. $R$-factors based on $F^{2}$ are statistically about twice as large as those based on $F$, and $R$ - factors based on ALL data will be even larger.

Fractional atomic coordinates and isotropic or equivalent isotropic displacement parameters $\left(\AA^{2}\right)$

\begin{tabular}{lllll}
\hline & $x$ & $y$ & $z$ & $U_{\text {iso }} * / U_{\text {eq }}$ \\
\hline C1 & $0.5886(2)$ & $0.25153(11)$ & $0.23432(10)$ & $0.0172(2)$ \\
C2 & $0.7514(2)$ & $0.14590(11)$ & $0.34545(10)$ & $0.0201(2)$ \\
H2A & 0.6664 & 0.1673 & 0.4289 & $0.024^{*}$ \\
H2B & 0.9522 & 0.1560 & 0.3350 & $0.024^{*}$ \\
C3 & $0.7394(2)$ & $-0.01067(12)$ & $0.34647(11)$ & $0.0264(3)$ \\
H3A & 0.8587 & -0.0815 & 0.4150 & $0.032^{*}$ \\
H3B & 0.5406 & -0.0233 & 0.3673 & $0.032^{*}$ \\
C4 & $0.8456(3)$ & $-0.04206(13)$ & $0.21466(12)$ & $0.0290(3)$ \\
H4A & 0.8225 & -0.1407 & 0.2159 & $0.035^{*}$
\end{tabular}


supporting information

$\begin{array}{lllll}\text { H4B } & 1.0513 & -0.0415 & 0.1989 & 0.035^{*} \\ \text { C5 } & 0.6822(2) & 0.07141(13) & 0.10418(11) & 0.0272(3) \\ \text { H5A } & 0.4801 & 0.0636 & 0.1148 & 0.033^{*} \\ \text { H5B } & 0.7631 & 0.0511 & 0.0199 & 0.033^{*} \\ \text { C6 } & 0.6986(2) & 0.22686(12) & 0.10284(10) & 0.0217(2) \\ \text { H6A } & 0.8983 & 0.2385 & 0.0843 & 0.026^{*} \\ \text { H6B } & 0.5815 & 0.2992 & 0.0343 & 0.026^{*} \\ \text { C7 } & -0.0539(2) & 0.51593(11) & 0.19148(10) & 0.0173(2) \\ \text { C8 } & -0.1182(2) & 0.51675(11) & 0.33402(10) & 0.0188(2) \\ \text { H8A } & -0.1150 & 0.4156 & 0.3873 & 0.023^{*} \\ \text { H8B } & 0.0320 & 0.5522 & 0.3629 & 0.023^{*} \\ \text { C9 } & -0.3962(2) & 0.61033(11) & 0.35514(10) & 0.0190(2) \\ \text { N1 } & 0.36168(17) & 0.33947(9) & 0.26408(8) & 0.0176(2) \\ \text { N2 } & 0.19288(17) & 0.42828(9) & 0.16325(8) & 0.0183(2) \\ \text { H1 } & 0.227(3) & 0.4221(14) & 0.0801(14) & 0.022^{*} \\ \text { N3 } & -0.61224(19) & 0.68227(10) & 0.37675(9) & 0.0251(2) \\ \text { O1 } & -0.21717(15) & 0.59183(8) & 0.10701(7) & 0.0228(2) \\ \end{array}$

Atomic displacement parameters $\left(\AA^{2}\right)$

\begin{tabular}{lllllll}
\hline & $U^{11}$ & $U^{22}$ & $U^{33}$ & $U^{12}$ & $U^{13}$ & $U^{23}$ \\
\hline C1 & $0.0182(4)$ & $0.0185(5)$ & $0.0157(5)$ & $-0.0059(4)$ & $-0.0009(4)$ & $-0.0038(4)$ \\
C2 & $0.0207(5)$ & $0.0225(5)$ & $0.0153(5)$ & $-0.0025(4)$ & $-0.0019(4)$ & $-0.0038(4)$ \\
C3 & $0.0310(6)$ & $0.0197(5)$ & $0.0229(6)$ & $-0.0032(4)$ & $0.0056(5)$ & $-0.0016(4)$ \\
C4 & $0.0347(6)$ & $0.0210(5)$ & $0.0309(6)$ & $-0.0078(4)$ & $0.0109(5)$ & $-0.0102(5)$ \\
C5 & $0.0217(5)$ & $0.0400(7)$ & $0.0266(6)$ & $-0.0090(5)$ & $0.0045(4)$ & $-0.0201(5)$ \\
C6 & $0.0179(5)$ & $0.0282(6)$ & $0.0150(5)$ & $-0.0001(4)$ & $0.0000(4)$ & $-0.0036(4)$ \\
C7 & $0.0189(5)$ & $0.0185(5)$ & $0.0151(5)$ & $-0.0058(4)$ & $0.0002(4)$ & $-0.0043(4)$ \\
C8 & $0.0192(5)$ & $0.0217(5)$ & $0.0155(5)$ & $-0.0036(4)$ & $-0.0009(4)$ & $-0.0057(4)$ \\
C9 & $0.0241(5)$ & $0.0199(5)$ & $0.0149(5)$ & $-0.0085(4)$ & $0.0000(4)$ & $-0.0050(4)$ \\
N1 & $0.0196(4)$ & $0.0185(4)$ & $0.0142(4)$ & $-0.0041(3)$ & $-0.0031(3)$ & $-0.0027(3)$ \\
N2 & $0.0196(4)$ & $0.0215(5)$ & $0.0118(4)$ & $-0.0018(3)$ & $-0.0011(3)$ & $-0.0034(3)$ \\
N3 & $0.0253(5)$ & $0.0255(5)$ & $0.0240(5)$ & $-0.0037(4)$ & $0.0020(4)$ & $-0.0087(4)$ \\
O1 & $0.0221(4)$ & $0.0263(4)$ & $0.0163(4)$ & $0.0009(3)$ & $-0.0027(3)$ & $-0.0043(3)$ \\
& & & & & & \\
\hline
\end{tabular}

Geometric parameters $\left(\AA,{ }^{\circ}\right)$

\begin{tabular}{llll}
\hline $\mathrm{C} 1-\mathrm{N} 1$ & $1.2845(13)$ & $\mathrm{C} 5-\mathrm{H} 5 \mathrm{~A}$ & 0.99 \\
$\mathrm{C} 1-\mathrm{C} 2$ & $1.5032(14)$ & $\mathrm{C} 5-\mathrm{H} 5 \mathrm{~B}$ & 0.99 \\
$\mathrm{C} 1-\mathrm{C} 6$ & $1.5036(14)$ & $\mathrm{C} 6-\mathrm{H} 6 \mathrm{~A}$ & 0.99 \\
$\mathrm{C} 2-\mathrm{C} 3$ & $1.5371(15)$ & $\mathrm{C} 6-\mathrm{H} 6 \mathrm{~B}$ & 0.99 \\
$\mathrm{C} 2-\mathrm{H} 2 \mathrm{~A}$ & 0.99 & $\mathrm{C} 7-\mathrm{O} 1$ & $1.2306(12)$ \\
$\mathrm{C} 2-\mathrm{H} 2 \mathrm{~B}$ & 0.99 & $\mathrm{C} 7-\mathrm{N} 2$ & $1.3442(13)$ \\
$\mathrm{C} 3-\mathrm{C} 4$ & $1.5269(16)$ & $\mathrm{C} 7-\mathrm{C} 8$ & $1.5209(14)$ \\
$\mathrm{C} 3-\mathrm{H} 3 \mathrm{~A}$ & 0.99 & $\mathrm{C} 8-\mathrm{C} 9$ & $1.4622(14)$ \\
$\mathrm{C} 3-\mathrm{H} 3 \mathrm{~B}$ & 0.99 & $\mathrm{C} 8-\mathrm{H} 8 \mathrm{~A}$ & 0.99 \\
$\mathrm{C} 4-\mathrm{C} 5$ & $1.5273(17)$ & $\mathrm{C} 8-\mathrm{H} 8 \mathrm{~B}$ & 0.99 \\
$\mathrm{C} 4-\mathrm{H} 4 \mathrm{~A}$ & 0.99 & $\mathrm{C} 9-\mathrm{N} 3$ & $1.1457(13)$
\end{tabular}




\begin{tabular}{|c|c|c|c|}
\hline $\mathrm{C} 4-\mathrm{H} 4 \mathrm{~B}$ & 0.99 & $\mathrm{~N} 1-\mathrm{N} 2$ & $1.3938(12)$ \\
\hline $\mathrm{C} 5-\mathrm{C} 6$ & $1.5314(16)$ & $\mathrm{N} 2-\mathrm{H} 1$ & $0.900(14)$ \\
\hline $\mathrm{N} 1-\mathrm{C} 1-\mathrm{C} 2$ & $116.83(9)$ & $\mathrm{C} 4-\mathrm{C} 5-\mathrm{H} 5 \mathrm{~B}$ & 109.4 \\
\hline $\mathrm{N} 1-\mathrm{C} 1-\mathrm{C} 6$ & $128.63(9)$ & $\mathrm{C} 6-\mathrm{C} 5-\mathrm{H} 5 \mathrm{~B}$ & 109.4 \\
\hline $\mathrm{C} 2-\mathrm{C} 1-\mathrm{C} 6$ & $114.13(8)$ & $\mathrm{H} 5 \mathrm{~A}-\mathrm{C} 5-\mathrm{H} 5 \mathrm{~B}$ & 108.0 \\
\hline $\mathrm{C} 1-\mathrm{C} 2-\mathrm{C} 3$ & $108.83(9)$ & $\mathrm{C} 1-\mathrm{C} 6-\mathrm{C} 5$ & $108.10(9)$ \\
\hline $\mathrm{C} 1-\mathrm{C} 2-\mathrm{H} 2 \mathrm{~A}$ & 109.9 & $\mathrm{C} 1-\mathrm{C} 6-\mathrm{H} 6 \mathrm{~A}$ & 110.1 \\
\hline $\mathrm{C} 3-\mathrm{C} 2-\mathrm{H} 2 \mathrm{~A}$ & 109.9 & $\mathrm{C} 5-\mathrm{C} 6-\mathrm{H} 6 \mathrm{~A}$ & 110.1 \\
\hline $\mathrm{C} 1-\mathrm{C} 2-\mathrm{H} 2 \mathrm{~B}$ & 109.9 & $\mathrm{C} 1-\mathrm{C} 6-\mathrm{H} 6 \mathrm{~B}$ & 110.1 \\
\hline $\mathrm{C} 3-\mathrm{C} 2-\mathrm{H} 2 \mathrm{~B}$ & 109.9 & $\mathrm{C} 5-\mathrm{C} 6-\mathrm{H} 6 \mathrm{~B}$ & 110.1 \\
\hline $\mathrm{H} 2 \mathrm{~A}-\mathrm{C} 2-\mathrm{H} 2 \mathrm{~B}$ & 108.3 & $\mathrm{H} 6 \mathrm{~A}-\mathrm{C} 6-\mathrm{H} 6 \mathrm{~B}$ & 108.4 \\
\hline $\mathrm{C} 4-\mathrm{C} 3-\mathrm{C} 2$ & $111.01(9)$ & $\mathrm{O} 1-\mathrm{C} 7-\mathrm{N} 2$ & $122.06(9)$ \\
\hline $\mathrm{C} 4-\mathrm{C} 3-\mathrm{H} 3 \mathrm{~A}$ & 109.4 & $\mathrm{O} 1-\mathrm{C} 7-\mathrm{C} 8$ & $121.97(9)$ \\
\hline $\mathrm{C} 2-\mathrm{C} 3-\mathrm{H} 3 \mathrm{~A}$ & 109.4 & $\mathrm{~N} 2-\mathrm{C} 7-\mathrm{C} 8$ & $115.97(9)$ \\
\hline $\mathrm{C} 4-\mathrm{C} 3-\mathrm{H} 3 \mathrm{~B}$ & 109.4 & $\mathrm{C} 9-\mathrm{C} 8-\mathrm{C} 7$ & $111.64(8)$ \\
\hline $\mathrm{C} 2-\mathrm{C} 3-\mathrm{H} 3 \mathrm{~B}$ & 109.4 & $\mathrm{C} 9-\mathrm{C} 8-\mathrm{H} 8 \mathrm{~A}$ & 109.3 \\
\hline $\mathrm{H} 3 \mathrm{~A}-\mathrm{C} 3-\mathrm{H} 3 \mathrm{~B}$ & 108.0 & $\mathrm{C} 7-\mathrm{C} 8-\mathrm{H} 8 \mathrm{~A}$ & 109.3 \\
\hline $\mathrm{C} 3-\mathrm{C} 4-\mathrm{C} 5$ & $111.44(9)$ & $\mathrm{C} 9-\mathrm{C} 8-\mathrm{H} 8 \mathrm{~B}$ & 109.3 \\
\hline $\mathrm{C} 3-\mathrm{C} 4-\mathrm{H} 4 \mathrm{~A}$ & 109.3 & $\mathrm{C} 7-\mathrm{C} 8-\mathrm{H} 8 \mathrm{~B}$ & 109.3 \\
\hline $\mathrm{C} 5-\mathrm{C} 4-\mathrm{H} 4 \mathrm{~A}$ & 109.3 & $\mathrm{H} 8 \mathrm{~A}-\mathrm{C} 8-\mathrm{H} 8 \mathrm{~B}$ & 108.0 \\
\hline $\mathrm{C} 3-\mathrm{C} 4-\mathrm{H} 4 \mathrm{~B}$ & 109.3 & $\mathrm{~N} 3-\mathrm{C} 9-\mathrm{C} 8$ & $177.32(11)$ \\
\hline $\mathrm{C} 5-\mathrm{C} 4-\mathrm{H} 4 \mathrm{~B}$ & 109.3 & $\mathrm{C} 1-\mathrm{N} 1-\mathrm{N} 2$ & $117.65(9)$ \\
\hline $\mathrm{H} 4 \mathrm{~A}-\mathrm{C} 4-\mathrm{H} 4 \mathrm{~B}$ & 108.0 & $\mathrm{C} 7-\mathrm{N} 2-\mathrm{N} 1$ & $119.30(9)$ \\
\hline $\mathrm{C} 4-\mathrm{C} 5-\mathrm{C} 6$ & $111.25(9)$ & $\mathrm{C} 7-\mathrm{N} 2-\mathrm{H} 1$ & $116.5(8)$ \\
\hline $\mathrm{C} 4-\mathrm{C} 5-\mathrm{H} 5 \mathrm{~A}$ & 109.4 & $\mathrm{~N} 1-\mathrm{N} 2-\mathrm{H} 1$ & $123.6(8)$ \\
\hline $\mathrm{C} 6-\mathrm{C} 5-\mathrm{H} 5 \mathrm{~A}$ & 109.4 & & \\
\hline $\mathrm{N} 1-\mathrm{C} 1-\mathrm{C} 2-\mathrm{C} 3$ & $114.56(10)$ & $\mathrm{O} 1-\mathrm{C} 7-\mathrm{C} 8-\mathrm{C} 9$ & $3.02(14)$ \\
\hline $\mathrm{C} 6-\mathrm{C} 1-\mathrm{C} 2-\mathrm{C} 3$ & $-58.72(11)$ & $\mathrm{N} 2-\mathrm{C} 7-\mathrm{C} 8-\mathrm{C} 9$ & $-177.51(9)$ \\
\hline $\mathrm{C} 1-\mathrm{C} 2-\mathrm{C} 3-\mathrm{C} 4$ & $54.68(12)$ & $\mathrm{C} 2-\mathrm{C} 1-\mathrm{N} 1-\mathrm{N} 2$ & $-173.88(8)$ \\
\hline $\mathrm{C} 2-\mathrm{C} 3-\mathrm{C} 4-\mathrm{C} 5$ & $-54.91(12)$ & $\mathrm{C} 6-\mathrm{C} 1-\mathrm{N} 1-\mathrm{N} 2$ & $-1.72(16)$ \\
\hline $\mathrm{C} 3-\mathrm{C} 4-\mathrm{C} 5-\mathrm{C} 6$ & $55.98(12)$ & $\mathrm{O} 1-\mathrm{C} 7-\mathrm{N} 2-\mathrm{N} 1$ & $-176.79(9)$ \\
\hline $\mathrm{N} 1-\mathrm{C} 1-\mathrm{C} 6-\mathrm{C} 5$ & $-113.10(12)$ & $\mathrm{C} 8-\mathrm{C} 7-\mathrm{N} 2-\mathrm{N} 1$ & $3.75(13)$ \\
\hline $\mathrm{C} 2-\mathrm{C} 1-\mathrm{C} 6-\mathrm{C} 5$ & $59.23(11)$ & $\mathrm{C} 1-\mathrm{N} 1-\mathrm{N} 2-\mathrm{C} 7$ & $176.44(9)$ \\
\hline $\mathrm{C} 4-\mathrm{C} 5-\mathrm{C} 6-\mathrm{C} 1$ & $-56.13(11)$ & & \\
\hline
\end{tabular}

Hydrogen-bond geometry $\left(\AA,{ }^{\circ}\right)$

\begin{tabular}{lllll}
\hline$D-\mathrm{H} \cdots A$ & $D-\mathrm{H}$ & $\mathrm{H} \cdots A$ & $D \cdots A$ & $D-\mathrm{H}^{\cdots} A A$ \\
\hline $\mathrm{N} 2-\mathrm{H} 1 \cdots \mathrm{O} 1^{\mathrm{i}}$ & $0.900(14)$ & $2.052(15)$ & $2.9399(12)$ & $168.4(11)$ \\
$\mathrm{C} 6-\mathrm{H} 6 B \cdots \mathrm{O} 1^{\mathrm{i}}$ & 0.99 & 2.32 & $3.2736(13)$ & 161 \\
$\mathrm{C} 8-\mathrm{H} 8 B \cdots \mathrm{N} 3^{\mathrm{ii}}$ & 0.99 & 2.41 & $3.3783(14)$ & 165 \\
\hline
\end{tabular}

Symmetry codes: (i) $-x,-y+1,-z$; (ii) $x+1, y, z$. 